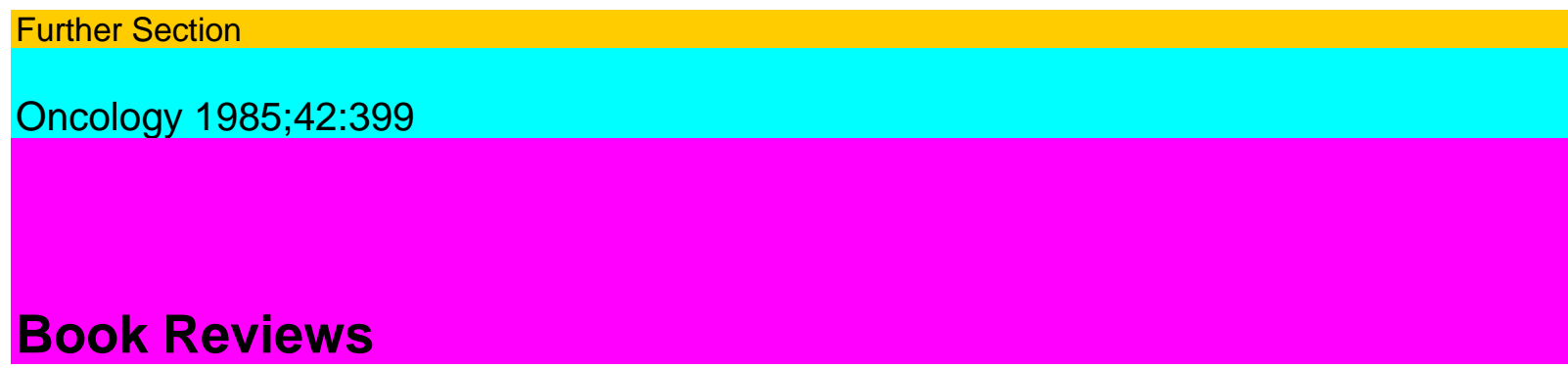

\title{
I. Besznyak
}

Mediastinal Tumors and Pseudotumors

Karger, Basel 1984

336 pp., SFr. 129.-/DM 154.-/USS 77.25

ISBN 3-8055-3582-1

The present book, written by experienced surgical and pathological specialists, is a comprehensive outline of mediastinal tumors. Diagnostics, pathology, clinics and therapy have been explained based on a large number of patients, following brief introductory remarks on general anatomy of the mediastinum as well as a chapter on symptomatology and estimation of the validity of diagnostic procedures, footing on a division into 11 chapters: tumors and cysts of the thymus, neurogenic, teratoid and cystic tumors of the mediastinum. The authors have also dealt with rare tumors and discussed differential-diagnostic problems of lymph node swelling. Important is the detailed discussion of aneurysms, the intrathoracal strumas and spatial requirements that may mimick the presence of a mediastinal tumor. The last chapter deals with special aspects of the surgical treatment, giving judgement on indications, contraindications and surgical incision procedures. The book has a clear diction, good arrangement and remarkably long lists of references at the end of each chapter. The latter will considerably facilitate further study of the subject. Regrettably, the authors - overdoing their modesty - have failed to give a detailed evaluation of their own patients (more than 600) in a separate chapter, which no doubt would have added to the book's value. The pictorial juxtaposition of the macroscopic preparation, histological picture and X-ray is instructive. What is left to be desired, however, is a better reproduction of the roentgenograms with more visibility of details.

The book being excellent in print and quality of paper can be warmly recommended to roentgenologists, pulmologists, pathologists and oncosurgeons alike.

P. Greiner

Jens Overgaard

Review Lectures, Symposium Summaries

and Workshop Summaries

Hyperthermic Oncology 1984, vol. 2 Taylor \& Francis, London 1985385 pp.; E 40.00, 2 vol. E 95.00 ISBN 0-85066-281-8

In these two large-sized volumes the Proceedings of the 4th International Symposium on Hyperthermic Oncology, held in Aar-hus July 2-4, 1984, are presented.

In the first volume summaries of more than 200 papers presented upon this occasion are given, the second volume is devoted to review lectures, summaries of symposia and workshops, in total another 30 papers in full length.

The abstract

s, mostly dealing with very specific investigations into a considerable number of topics, are put together in the chapters: mechanisms of heat injury; heat shock proteins - mechanisms of thermotolerance; environmental and vascular effects; thermal 
dose, thermotolerance - implications for clinical treatment; whole body hyperthermia biological and clinical studies; hyperthermia and radiation - biological and clinical studies; hyperthermia and drugs - biological and clinical studies; immunology and transformation; thermal modeling; thermometry; interstitial techniques; regional hyperthermia and heating of deep-seated tumors; surface applicators and heating systems; brain, gynecological, urogenital and gastrointestinal tumors; other clinical and biological studies.

The great amount of technical problems and of thermometry, rising questions concerning the combination of hyperthermia with other methods, as well as the variety of data presented lead to the result that - having gone thoroughly through the books - there are rather more questions left open than answered. The fact that the title 'Hyperthermia - the Next Stage in the Oncologist's Odyssey' has been chosen by the last speaker serves as a confirmation of the above-said. For anybody not closely familiar with the problems of hyperthermia the way to information from these two volumes is a rather hard one. For anybody interested in hyperthermia in oncology the books will certainly provide the basis of all knowledge presently available.

H. Wrba, Vienna

H.G. Bender, L. Beck Cancer of the Uterine Cervix

Cancer Campaign, vol. 8 Fischer, Stuttgart 1985 VIII+ 231 pp.; DM 110.-ISBN 3-437-10847-6

The book contains the proceedings of the XVth Symposium of the 'Gesellschaft zur

Bekampfung der Krebskrankheiten Nord-rhein-Westfalen e.V.’. Contributions are focussed on various aspects of the biological, epidemiological and methodological background for controlling cervical cancer by means of mass-screening programmes.

Thus, the first part of papers presents results of screening programmes in Canada, Finland and the Federal Republic of Germany and gives some theoretical considerations resulting in mathematical models for cervical cancer screening. A number of further contributions offer a survey about some results of studies interpreting the natural history of cervical cancer and investigating factors possibly influencing it. Other presentations provide information and results of therapeutic procedures for cervical intraepithelial neoplasias.

$\mathrm{T}$. The editors succeeded in bringing together a considerable number of scientists wellexperienced in this field. This results in a sufficient insight in many aspects linked with cervical cancer control. But because the symposium was already held in July 1983

the experienced reader will miss a considerable amount of scientific results obtained meanwhile. This concerns mostly latest data about the protective effect of pap smear obtained by means of case-control approaches and more detailed investigations regarding the possible aetiological role of human papilloma virus infections for cervical cancer. Nevertheless

the book can be recommended to all those who are interested in cervical cancer control.

K. Ebeling

Berlin-Buch 